\title{
Mensuração da satisfação por meio da adaptação de um modelo hierárquico baseado na lógica fuzzy
}

Karine Dutra Viana ${ }^{1}$

Viviane Leite de Dias Mattos ${ }^{1}$

\begin{abstract}
Resumo: O presente trabalho trata de um modelo de análise hierárquica, fundamentado na lógica fuzzy, denominado COPPE-Cosenza. Inicialmente este modelo foi desenvolvido para análise de zoneamento industrial. Além de destacar brevemente sua metodologia, o objetivo deste estudo é propor algumas adaptações e mostrar o seu desempenho na mensuração da satisfação. Os dados utilizados são parte integrante de uma pesquisa que construiu uma escala para medir a satisfação de usuários de transporte público de uma determinada região. A análise desenvolvida permite estabelecer uma hierarquia entre os três indivíduos analisados a partir de sua satisfação. Embora nem todos os escores encontrados para a satisfação tenham sido iguais, houve concordância entre as hierarquias estabelecidas.
\end{abstract}

Palavras-chave: Análise da satisfação. Lógica fuzzy. Modelo hierárquico.

\begin{abstract}
This paper talks about a model of hierarchical analysis called COPPE-Cosenza. It is based on fuzzy logic and it was developed initially for the analysis of industrial zoning. In addition to focus briefly the methodology of model, the main objective is to propose some adjustments and show the performance of model for the analysis of the variable satisfaction. The data used are part of a research previously validated that examined the satisfaction of users of public transport in a given region. The analysis developed allows establishing a hierarchy among the three individuals analyzed from their satisfaction. Although not all the scores found for satisfaction were equal, there was agreement between established hierarchies.
\end{abstract}

Keywords: .Fuzzy Logic. Hierarchical model. Satisfaction analyses.

\section{Introdução}

Modelos hierárquicos são fundamentais em processos de tomada de decisão, pois permitem fácil compreensão e melhor avaliação de problemas que geralmente são complexos. A maioria dos métodos de análise hierárquica se utiliza da lógica booleana, no entanto, considera-se que quanto mais complexas e incertas são as variáveis envolvidas, mais adequada é a utilização da lógica fuzzy. Os dados obtidos em uma pesquisa com este tipo de variável geralmente são coletados por meio de observação ou levantamento de informações através de entrevistas e questionários, logo as informações obtidas podem ser subjetivas, visto que dependem de respostas de indivíduos que pensam e agem de maneira distinta.

A lógica fuzzy pode ser utilizada para lidar com certas variáveis utilizadas em nosso cotidiano, transmitidas e perfeitamente compreendidas linguisticamente, mas que tem invariavelmente permanecido fora do tratamento matemático tradicional.

As primeiras noções da lógica dos conceitos "vagos" foi desenvolvida pelo lógico polonês Jan Lukasiewicz, em 1920, que introduziu conjuntos com graus de pertinência $0,1 / 2 \mathrm{e} 1$. Mais tarde, esse grau de pertinência foi expandido para um número infinito de valores entre 0 e 1 , que hoje caracteriza a lógica fuzzy. Isto

\footnotetext{
${ }^{1}$ Programa de Pós-Graduação em Modelagem Computacional, FURG, Campus Carreiros - Av. Itália Km 8 - Rio Grande (RS) - Brasil

http://dx.doi.org/10.5335/rbca.v9i1.6694 
ocorreu por volta de 1965 quando o matemático, engenheiro e professor Loffi Asker Zadeh, com base nos conceitos da teoria dos conjuntos fuzzy, propôs a lógica fuzzy combinando os conceitos da lógica clássica e dos conjuntos de Lukasiewicz, definindo graus de pertinência. A lógica fuzzy ou lógica difusa é capaz de capturar informações imprecisas descritas em uma linguagem natural, como por exemplo, os conceitos de alto, bonito, maior, satisfeito, rápido, e transformá-los em valores numéricos, com capacidade de aceitar modos de raciocínios aproximados e não exatos.

A definição de algumas classes que expressam objetos da realidade não tem precisão no trato matemático tradicional. Como exemplo, [8] coloca que a classe de animais claramente inclui como membros cães, cavalos, aves etc. e obviamente exclui as rochas, fluidos, plantas, etc. No entanto, considerando, por exemplo, as estrelas do mar, as bactérias, etc. é incerto ou ambíguo as suas inclusões na classe dos animais. Do mesmo modo há ambiguidade quando se refere, por exemplo, ao "número 10" em relação a "números reais muito maiores que 1", ou classes de "mulheres bonitas" ou "homens altos":estas classes não constituem conjuntos matemáticos no sentido habitual do termos. O fato é que estas classes desempenham um papel importante no pensamento humano, em particular nos domínios de reconhecimento de padrões, comunicação de informações e abstração. Diferentemente da lógica clássica, que assume somente as posições de verdadeiro ou falso, sim ou não, zero ou um, a lógica fuzzy permite a existência de infinitas posições entre o verdadeiro e o falso, entre o sim e o não, entre o zero e o um.

O modelo aqui estudado é um modelo de análise hierárquica, denominado COPPE-Cosenza. Este foi concebido inicialmente para estudos de localização industrial de forma hierárquica e multicriteriosa, sendo basicamente executado em uma lógica de confronto entre demanda industrial e oferta de fatores de localização geográfica. Utiliza-se da lógica fuzzy e tem como principal função ser um apoio à tomada de decisão.

Levando em consideração a eficácia comprovada em várias aplicações, a simplicidade do modelo COPPE-Cosenza e ainda, tendo ciência da importância da lógica fuzzy no manuseio de variáveis como a satisfação, o objetivo deste trabalho é, a partir de uma análise crítica, tanto sob o ponto de vista teórico como prático, apresentar algumas adaptações da metodologia do modelo visando adequá-lo para mensurar a satisfação de clientes, comparando, de forma breve, o resultado encontrado em cada uma das propostas.

$\mathrm{Na}$ literatura estudada não foram encontradas aplicações do modelo COPPE-Cosenza para a análise específica de uma variável como a satisfação em relação à prestação de um determinado serviço. Assim, a adaptação do modelo para análise deste tipo de variável, comprovando sua funcionalidade, pode ser de considerável importância em estudos empresariais, na área da qualidade, assim como, talvez possa servir para mensurar outras variáveis, como a própria qualidade, motivação e desempenho, em uma perspectiva de avaliação de clientes e/ou empresas, pois se trata de um modelo simples e que apresenta grande eficiência em estudos já validados.

\section{O modelo Coppe-Cozenza de análise hierárquica}

Segundo [3], este modelo teve como inspiração o Modelo MASTERLI, 'Modelo di Assetto Territoriable e Localizzazione Industriale', publicado em 1974. Em 1975, a COPPE/UFRJ conjuntamente a duas empresas desenvolveu os primeiros modelos de localização no Brasil, quando então, Carlos Alberto Nunes Cosenza veio a complementar o modelo Masterli com modificações estruturais e operacionais que, conforme [4], possibilitaram operações com micro-regiões para localização industrial, com maior detalhamento dos perfis de oferta e demanda. Estas modificações consideram situações intermediárias que anteriormente estavam agrupadas em apenas dois níveis, liberdade para ponderar eventual excesso de oferta de fatores locacionais e liberdade para penalizar eventual escassez de oferta destes mesmos fatores.

Inicialmente este modelo era operacionalizado apenas com a lógica clássica, porém no ano de 1981, a lógica fuzzy com suas variáveis linguísticas foi introduzida no modelo. Sua metodologia consiste basicamente na interação entre duas matrizes, uma representativa da demanda de fatores necessários a um determinado projeto e a outra de oferta destes fatores. Para a definição destas duas matrizes são definidos dois conjuntos fuzzy, um deles representando a demanda e o outro, a oferta.

Sejam $A=\left(a_{i j}\right)_{h x n}$, a matriz representante da demanda de $h$ atividades relativamente à $n$ fatores/atributos e $B=\left(b_{j k}\right)_{n x m}$, a matriz representante da oferta de $n$ fatores/atributos por $m$ alternativas.

Revista Brasileira de Computação Aplicada(ISSN 2176-6649), Passo Fundo, v. 9, n. 1, p. 52-62, abr. 201753 
Seja $F=\{f i \mid 1, \ldots, n\}$, um conjunto finito de fatores/atributos denotado genericamente por $f$. Então, o conjunto fuzzy $\tilde{A}$ em $f$ é um conjunto de pares ordenados $\tilde{A}=\left\{\left(f, \mu_{\tilde{A}}(f)\right) \mid f \in F\right\}$, onde $\tilde{A}$ é a representação fuzzy da matriz demanda e $\mu_{\tilde{A}}(f)$ é a função de pertinência representando o grau de importância dos fatores.

Do mesmo modo, tem-se $\widetilde{B}=\left\{\left(f, \mu_{\tilde{B}}(f)\right) \mid f \in F\right\}$, onde $\widetilde{B}$ é a representação fuzzy da matriz oferta e $\mu_{\tilde{B}}(f)$ é a função de pertinência que representa o grau de atendimento dos fatores disponibilizados pelas diversas alternativas. $\tilde{A}=\left\{a_{i} \mid i=1, \ldots, m\right\}$, é o conjunto de fatores demandados por diferentes tipos de projetos que formam a matriz $\tilde{A}$ e $B=\left\{b_{k} \mid k=1, \ldots, n\right\}$, o conjunto de alternativas onde está contido $F=\left\{f_{k} \mid k=1, \ldots, n\right\}$, o conjunto de fatores comuns aos vários projetos que formam a matriz $\tilde{B}$.

No intervalo $[0,1]$ estão incluídos todos os valores de suporte de $\tilde{A}$ e $\tilde{B}$, identificados como variáveis linguísticas, e exemplificados na Tabela 1.

Tabela1: Variáveis linguísticas para $\tilde{a}_{i j}$ e $\tilde{b}_{j k}$

\begin{tabular}{ccccc}
\hline & \multicolumn{2}{c}{$b_{j k}$} & & $a_{i j}$ \\
FATORES & & Graus para as alternativas $k_{i}$ & & Importância para o projeto \\
& $B_{1}$ & $B_{2}$ & $B_{3}$ & \\
\hline$f_{1}$ & Fraco & Fraco & Superior & Condicionante \\
$f_{2}$ & Fraco & Superior & Bom & Crítico \\
$f_{3}$ & Bom & Superior & Regular & Irrelevante \\
$\ldots$ & $\ldots$ & $\ldots$ & $\ldots$ & $\ldots$ \\
\hline
\end{tabular}

Fonte: Adaptado de [6].

Obs.: $B_{1}, B_{2}, \ldots, B_{m}=$ alternativas; $\tilde{a}_{i j}=$ coeficiente do grau de importância do fator $j$ em relação ao projeto $i ; \tilde{b}_{j k}$ $=$ coeficiente resultante do nível do fator $j$ disponível na área $k$.

Com o objetivo de contornar o problema da distância assimétrica e aumentar a precisão do modelo, consideram-se dois elementos genéricos, $\tilde{a}_{i j}$ e $\tilde{b}_{j k}$, pertencentes à $\tilde{A}$ e $\tilde{B}$, respectivamente. O produto entre eles será obtido através de um operador do tipo $\tilde{a}_{i j} \otimes \tilde{b}_{j k}=\left(\tilde{c}_{i k}\right)_{h x m}$. Esta operação terá como resultado uma matriz de possibilidades representativa do agregado das comparações de demanda /oferta de cada fator. A operação deve proceder conforme a Tabela 2.

Tabela 2: Produto $\tilde{A} \otimes \tilde{B}$

\begin{tabular}{ccccc}
\hline Requerimento de fatores & \multicolumn{3}{c}{ Disponibilidade de fatores } \\
$\mathrm{a}_{\mathrm{ij}} \otimes \mathrm{b}_{\mathrm{jk}}$ & 0 & $\ldots$ & 1 \\
\hline 0 & $0^{+}$ & & $0^{++}$ \\
$\ldots$ & & & 1 & \\
1 & 0 & & 1 \\
\hline
\end{tabular}

Fonte: Adaptado de [7].

Obs.: $0^{+}=1 / n ! ; \quad 0^{++}=1 / n ; n=$ número de fatores considerados.

Para [2], este modelo tem como maior utilidade a tomada de decisão entre diferentes perfis de diferentes graus de importância aos fatores gerais e específicos com elevado número de alternativas. Ele já foi utilizado para estudos de empreendimentos e atividades que visam atender ações sustentáveis e inovações tecnológicas. No entanto, até o presente momento não foram encontradas aplicações para avaliação de variáveis como a satisfação. A ideia aqui apresentada está concentrada exatamente neste ponto: avaliar a possibilidade de utilização do modelo COPPE-Cosenza, com algumas adaptações, na mensuração da satisfação de clientes, já que se trata de um problema que depende de diferentes opiniões com vários níveis de importância considerados por cada respondente e análises de fatores que concordem com o objeto a ser avaliado.

Para definir a distância entre os elementos das duas matrizes existem vários operadores. Entretanto, eles foram criados para serem utilizados em uma determinada realidade. Dentre as regras operacionais já existentes, 
têm-se a Matriz Diagonal Superior, a Matriz de Diferença de Pertinências e a Matriz Relacionamento de Pertinências, apresentadas nas Tabelas 3, 4 e 5, respectivamente.

Tabela 3: Matriz diagonal inferior

\begin{tabular}{ccccc}
\hline $\begin{array}{c}\text { Demanda por } \\
\text { fatores } \\
\mathrm{a}_{\mathrm{ij}} \otimes \mathrm{b}_{\mathrm{ik}}\end{array}$ & $\mathrm{A}$ & $\mathrm{B}$ & $\mathrm{C}$ & $\mathrm{D}$ \\
\hline $\mathrm{A}$ & 1 & 0 & 0 & 0 \\
$\mathrm{~B}$ & $1+1 / n$ & 1 & 0 & 0 \\
$\mathrm{C}$ & $1+2 / n$ & $1+1 / n$ & 1 & 0 \\
$\mathrm{D}$ & $1+3 / n$ & $1+2 / n$ & $1+1 / n$ & 1 \\
\hline
\end{tabular}

Fonte: Adaptada de [6].

Obs.: $n=$ número de fatores considerados.

Tabela 4: Matriz de diferença de pertinências

\begin{tabular}{|c|c|c|c|}
\hline $\begin{array}{c}\text { Demanda por } \\
\text { fatores }\end{array}$ & & Ofertas $\mathrm{p}$ & \\
\hline$a_{i j} \otimes b_{j k}$ & 0 & $\mu B_{i}(x)$ & 1 \\
\hline 0 & $0^{+}$ & . & $0^{++}$ \\
\hline$\ldots$ & & 1 & $1+[\mu \mathrm{B}(\mathrm{x})-\mu \mathrm{A}(\mathrm{x})]$ \\
\hline$\mu \mathrm{A}_{\mathrm{j}}(\mathrm{x})$ & & 1 & \\
\hline ... & $1+[\mu \mathrm{B}(\mathrm{x})-\mu \mathrm{A}(\mathrm{x})]$ & & \\
\hline 1 & 0 & . & 1 \\
\hline
\end{tabular}

Fonte: Adaptada de [6].

Obs.: $\mu A_{x}(x)=$ grau de pertinência associado a cada classe para a demanda; $\mu B_{x}(x)=$ grau de pertinência associado a cada classe para a oferta.

Tabela 5: Matriz Relacionamento de Pertinências

\begin{tabular}{|c|c|c|c|c|c|}
\hline \multicolumn{3}{|c|}{ Demanda por fatores } & \multicolumn{3}{|c|}{ Ofertas por fatores } \\
\hline$a_{i j} \otimes b_{j k}$ & 0 & $\mu \mathrm{B}_{1}(\mathrm{x})$ & $\mu \mathrm{B}_{2}(\mathrm{x})$ & $\mu \mathrm{B}_{3}(\mathrm{x})$ & $\mu \mathrm{B}_{4}(\mathrm{x})$ \\
\hline & 1 & 1 & 1 & 1 & 1 \\
\hline $\mathbf{0}$ & $\overline{\mathrm{n} !}$ & $\overline{\mathrm{n}-3}$ & $\overline{n-2}$ & $\overline{n-1}$ & $\bar{n}$ \\
\hline$\mu \mathbf{A}_{1}(\mathbf{x})$ & 0 & 1 & $1+\frac{\mu B_{1}(x)}{n}$ & $1+\frac{\mu B_{2}(x)}{n}$ & $1+\frac{\mu B_{3}(x)}{n}$ \\
\hline $\boldsymbol{\mu} \mathbf{A}_{2}(\mathbf{x})$ & 0 & $\frac{\mu B_{1}(x)}{\mu A_{2}(x)}$ & 1 & $1+\frac{\mu B_{1}(x)}{n}$ & $1+\frac{\mu B_{2}(x)}{n}$ \\
\hline $\boldsymbol{\mu} \mathbf{A}_{\mathbf{3}}(\mathbf{x})$ & 0 & $\frac{\mu B_{1}(x)}{\mu A_{3}(x)}$ & $\frac{\mu B_{2}(x)}{\mu A_{3}(x)}$ & 1 & $1+\frac{\mu B_{3}(x)}{n}$ \\
\hline $\boldsymbol{\mu} \mathbf{A}_{4}(\mathbf{x})$ & 0 & $\frac{\mu B_{1}(x)}{\mu A_{4}(x)}$ & $\frac{\mu B_{2}(x)}{\mu A_{4}(x)}$ & $\frac{\mu B_{3}(x)}{\mu A_{4}(x)}$ & 1 \\
\hline
\end{tabular}

Fonte: Adaptado de [6]. 
Obs.: $\mu A x(x)=$ grau de pertinência associado a cada classe para a demanda; $\mu B x(x)=$ grau de pertinência associado a cada classe para a oferta.

\section{Metodologia}

Partindo da proposta deste trabalho, que se concentra em analisar a satisfação, utilizou-se um instrumento e um banco de dados pertencentes a uma pesquisa realizada na Universidade Federal do Rio Grande, no qual a mensuração foi fundamentada na lógica fuzzy. O referido estudo baseou-se em estudos anteriores sobre a avaliação de transporte público, em opiniões de especialistas e levantamento de dados.

Nesta pesquisa foram utilizadas técnicas qualitativas, que se concretizaram através entrevistas e questionários, e quantitativas, utilizando análise fatorial e avaliação da confiabilidade, sendo a mensuração da satisfação feita por meio de controladores fuzzy. O modelo baseou-se em quatro dimensões do construto: atendimento, local de embarque/desembarque, veículo e segurança, avaliadas por vinte e um itens. Este instrumento é considerado válido e fidedigno para avaliar a satisfação de usuários com o sistema de transporte público e pode ser encontrado em [5].

A proposta para avaliação do Modelo COPPE-Cosenza na mensuração da satisfação utilizou os dados de uma pequena amostra selecionada aleatoriamente no banco de dados supracitado, sendo verificado o desempenho das adaptações sugeridas nesta nova proposta. As matrizes de demanda e oferta de fatores tomaram a forma das apresentadas nas Tabelas 6 e 7.

Tabela 6: Importância avaliada atribuída a cada item

\begin{tabular}{|c|c|c|c|c|c|c|}
\hline & Item 1 & Item 2 & $\ldots$ & Item $j$ & $\ldots$ & Item $n$ \\
\hline Indivíduo 1 & $a_{11}$ & $a_{12}$ & $\cdots$ & $a_{1 j}$ & $\cdots$ & $a_{1 n}$ \\
\hline Indivíduo 2 & $a_{21}$ & $a_{22}$ & $\cdots$ & $a_{2 j}$ & $\cdots$ & $a_{2 n}$ \\
\hline$\cdots$ & $\cdots$ & $\cdots$ & $\cdots$ & $\cdots$ & $\cdots$ & $\cdots$ \\
\hline Indivíduo $i$ & $a_{i 1}$ & $a_{i 2}$ & $\ldots$ & $a_{i j}$ & $\cdots$ & $a_{i n}$ \\
\hline$\cdots$ & $\cdots$ & $\cdots$ & $\cdots$ & $\cdots$ & $\cdots$ & $\cdots$ \\
\hline Indivíduo $m$ & $a_{m 1}$ & $a_{m 2}$ & $\ldots$ & $a_{m j}$ & $\ldots$ & $a_{m n}$ \\
\hline
\end{tabular}

Fonte: as autoras.

Obs.: $a_{i j}=$ coeficiente fuzzy (grau de importância) do item $j$ para a pessoa $i$.

Tabela 7: Percepção de oferta por item

\begin{tabular}{|c|c|c|c|c|c|c|}
\hline & Indivíduo 1 & Indivíduo2 & $\ldots$ & Indivíduo $k$ & $\ldots$ & Indivíduo $m$ \\
\hline Item 1 & $b_{11}$ & $b_{12}$ & $\ldots$ & $b_{1 k}$ & $\ldots$ & $b_{1 m}$ \\
\hline Item 2 & $b_{21}$ & $b_{22}$ & $\ldots$ & $b_{2 k}$ & $\ldots$ & $b_{2 m}$ \\
\hline ... & $\ldots$ & $\ldots$ & $\ldots$ & $\ldots$ & $\ldots$ & $\ldots$ \\
\hline Item $j$ & $b_{j 1}$ & $b_{j 2}$ & $\ldots$ & $b_{j k}$ & $\ldots$ & $b_{i m}$ \\
\hline$\ldots$ & $\ldots$ & $\ldots$ & $\ldots$ & $\ldots$ & $\ldots$ & $\ldots$ \\
\hline Item $n$ & $b_{n 1}$ & $b_{n 2}$ & $\ldots$ & $b_{n k}$ & $\ldots$ & $b_{n m}$ \\
\hline
\end{tabular}

Fonte: as autoras.

Obs.: $\mathrm{b}_{\mathrm{ij}}=$ coeficiente fuzzy da pessoa $k$ em relação ao item $j$.

De acordo com [5], os itens são as características a serem avaliadas individualmente em termos de importância (demanda) e qualidade do serviço oferecido (oferta), fornecendo uma mensuração para a satisfação. Neste estudo a intenção é comparar a importância de cada item para a satisfação e a qualidade do que é disponibilizado de acordo com o que o indivíduo consegue perceber, medindo-se a distância entre a importância 
e a oferta deste item. Entre os itens julgados necessários para mensurar a satisfação estão tempo de viagem, preços da passagem, tempo de espera e conservação do veículo, entre outros.

Dos pontos observados na metodologia original do modelo COPPE-Cosenza, que mereceram maior atenção em relação a possíveis adaptações, destacam-se as operações de cotejo que definem a distância entre os valores de demanda e oferta, que são apresentadas nas Tabelas 3, 4 e 5. A proposta manteve-se em torno de obter um operador que fosse suficientemente capaz de entender a variável analisada, a satisfação. Para tais adaptações foram utilizadas como base as Tabelas 4 e 5 . Não foi utilizada a Tabela 3 por não utilizar graus de pertinência, mas apenas o número total de fatores da análise, o que foi considerado um processo não fuzzy. As adaptações realizadas partiram das regras apresentadas a seguir.

I. Foram considerados 10 níveis para o suporte do conjunto fuzzy que mede a satisfação, pois quanto menor for o intervalo de classes, mais abrangente serão os resultados possíveis na relação demanda e oferta. Estas classes estão divididas e associadas a um grau de pertinência da seguinte forma:

classe 1: $00 \vdash 10$ com grau de pertinência 0,1 ;

classe 2: $10 \vdash 20$ com grau de pertinência 0,2 ; classe 3: $20 \vdash 30$ com grau de pertinência 0,3 ; classe 4: $30 \vdash 40$ com grau de pertinência 0,4 ; classe 5: $40+50$ com grau de pertinência 0,5 ; classe 6: $50 \vdash 60$ com grau de pertinência 0,6; classe 7: $60+70$ com grau de pertinência 0,7; classe 8: $70 \vdash 80$ com grau de pertinência 0,8 ; classe 9: $80 \vdash 90$ com grau de pertinência 0,9 ; classe 10: $90 \mathrm{H} 100$ com grau de pertinência 1,0 .

II. Sempre que um fator tem qualidade (oferta) maior ou igual à importância (demanda) a relação entre as duas posições deve resultar no valor 1 (um), ou seja, o indivíduo deve ser considerado muito satisfeito, atingindo o nível máximo de satisfação. Mesmo que o fator analisado disponha de mais do que o necessário, não se consideram excessos, ou seja, valores maiores que o valor 1 (um), pois entende-se que uma pontuação superior a este valor não influenciaria no resultado final da satisfação.

III. Se um fator tem disponibilidade (oferta) menor que a importância (demanda), o resultado entre as duas posições decresce gradativamente, variando de 0 à 0,9 , de acordo o nível de disponibilidade verificado.

Utilizando-se de tais considerações, foram reconstruídos os operadores, que resultaram nas matrizes apresentadas nas Tabelas 8 e 9 .

Tabela 8: Matriz diferença de Pertinências Adaptada

\begin{tabular}{|c|c|c|c|c|c|c|c|c|c|c|}
\hline $\begin{array}{c}\text { Demanda } \\
\text { por } \\
\text { Fatores }\end{array}$ & \multicolumn{10}{|c|}{ Oferta de fatores } \\
\hline$a_{i j} \times b_{j k}$ & $\mu B_{I}(x)$ & $\mu B_{2}(x)$ & $\mu B_{3}(x)$ & $\mu B_{4}(x$ & $\mu B_{5}(x$ & $\mu B_{6}(x$ & $\mu B_{7}(x$ & $\mu B_{8}(x$ & $\mu B_{9}(x$ & $\mu B_{10}(x)$ \\
\hline & & & & ) & ) & ) & ) & ) & ) & \\
\hline$\mu A_{1}(x)$ & 1 & 1 & 1 & 1 & 1 & 1 & 1 & 1 & 1 & 1 \\
\hline$\mu A_{2}(x)$ & & 1 & 1 & 1 & 1 & 1 & 1 & 1 & 1 & 1 \\
\hline$\mu A_{3}(x)$ & $\ldots$ & & 1 & 1 & 1 & 1 & 1 & 1 & 1 & 1 \\
\hline$\mu A_{4}(x)$ & & & & 1 & 1 & 1 & 1 & 1 & 1 & 1 \\
\hline$\mu A_{5}(x)$ & & & & & 1 & 1 & 1 & 1 & 1 & 1 \\
\hline$\mu A_{6}(x)$ & & & & & & 1 & 1 & 1 & 1 & 1 \\
\hline$\mu A_{7}(x)$ & & $1+[\mu B x$ & $A x(x)]$ & & & & 1 & 1 & 1 & 1 \\
\hline$\mu A_{8}(x)$ & & & & & & & & 1 & 1 & 1 \\
\hline$\mu A_{9}(x)$ & & & & & & & & & 1 & 1 \\
\hline$\mu A_{10}(x)$ & & & & & & & . & & & 1 \\
\hline
\end{tabular}

Fonte: as autoras.

Obs.: $\mu A_{x}(x)=$ grau de pertinência associado a cada classe para a demanda; $\mu B_{x}(x)=$ grau de pertinência associado a cada classe para oferta. 
Tabela 9: Matriz Relacionamento de Pertinências Adaptada

\begin{tabular}{|c|c|c|c|c|c|c|c|c|c|c|}
\hline $\begin{array}{c}\text { Demanda } \\
\text { por }\end{array}$ & & & & & Oferta de & fatores & & & & \\
\hline$a_{i j} \times b_{j j}$ & $\mu B_{l}(x)$ & $\mu B_{2}(x)$ & $\mu B_{3}(x)$ & $\mu B_{4}(x)$ & $\mu B_{5}(x)$ & $\mu B_{6}(x)$ & $\mu B_{7}(x)$ & $\mu B_{8}(x)$ & $\mu B_{9}(x)$ & $\mu B_{10}(x)$ \\
\hline$\mu \mathrm{A}_{1}(\mathrm{x})$ & 1 & 1 & 1 & 1 & 1 & 1 & 1 & 1 & 1 & 1 \\
\hline$\mu \mathrm{A}_{2}(\mathrm{x})$ & & 1 & 1 & 1 & 1 & 1 & 1 & 1 & 1 & 1 \\
\hline$\mu \mathrm{A}_{3}(\mathrm{x})$ & $\ldots$ & & 1 & 1 & 1 & 1 & 1 & 1 & 1 & 1 \\
\hline$\mu \mathrm{A}_{4}(\mathrm{x})$ & & & & 1 & 1 & 1 & 1 & 1 & 1 & 1 \\
\hline$\mu \mathrm{A}_{5}(\mathrm{x})$ & & & & & 1 & 1 & 1 & 1 & 1 & 1 \\
\hline$\mu \mathrm{A}_{6}(\mathrm{x})$ & & $\mu B x / \mu A x$ & & & & 1 & 1 & 1 & 1 & 1 \\
\hline$\mu \mathrm{A}_{7}(\mathrm{x})$ & & & & & & & 1 & 1 & 1 & 1 \\
\hline$\mu \mathrm{A}_{8}(\mathrm{x})$ & & & & & & & & 1 & 1 & 1 \\
\hline$\mu \mathrm{A}_{9}(\mathrm{x})$ & & & & & & & & & 1 & 1 \\
\hline$\mu \mathrm{A}_{10}(\mathrm{x})$ & $\ldots$ & & & & & & $\ldots$ & & & 1 \\
\hline
\end{tabular}

Fonte: as autoras.

Obs.: $\mu A_{x}(x)$ o grau de pertinência associado a cada classe para a demanda e $\mu B_{x}(x)$ o grau de pertinência associado a cada classe para oferta.

Para esta análise, foram selecionados no banco de dados 3 respondentes do instrumento que é composto por 21 itens. Estes itens são agrupados em 4 fatores: o primeiro fator composto por dez itens, o segundo fator por quatro itens, o terceiro por quatro itens e o quarto por dois itens. Em [5], para chegar a um valor final para cada fator, utilizaram-se os controladores fuzzy.

Conforme [1], é esperado que especialistas encontrem dificuldades em representar um conhecimento através de números reais. Em alguns casos, é comum que esta representação seja atribuída por diversos especialistas, dentre os quais há diversos julgamentos, aumentando a complexidade do problema. Uma possibilidade para representar numericamente uma determinada situação é a utilização dos controladores fuzzy, geralmente utilizados em problemas onde há imprecisão. Eles tem a capacidade de armazenamento de memória, permitindo decidir dentre relações diversas em um determinado problema e chegar a um valor mais redundante $\mathrm{e}$ coerente com a realidade, proporcionando melhores resultados para com o projeto em questão.

Os controladores fuzzy são constituídos de três etapas: a fuzzificação, a inferência e a defuzzificação. Como variáveis de entrada eles utilizam um universo crisp, que no processo de fuzzificação se transformam em números fuzzy. Através do processo de inferência, da base de regras e da defuzzificação, obtém-se uma saída também crisp resultante da interação entre os vários itens analisados.

O processo de fuzzificação (entradas discretas) consiste em realizar um mapeamento entre os valores numéricos das variáveis de entrada do controlador para graus de compatibilidade com conceitos linguísticos, ou seja, nesta etapa definem-se os termos linguísticos e suas respectivas funções de pertinência. Assim, os valores discretos obtidos na avaliação tornam-se valores fuzzy com diferentes graus de pertinência. As funções de pertinência mais utilizadas são as funções trapezoidais e triangulares.

Neste projeto, utilizou-se a função triangular, que é o modelo mais simples e mais utilizado em processos computacionais, representada conforme Equação (1).

$$
f(x)=\max \left(\min \left(\frac{x-a}{b-a}, \frac{c-x}{c-b}\right), 0\right)
$$

Onde, $a$ e $c$ são os extremos do suporte do conjunto fuzzy e $b$ é o seu core.

A base de regras deve conter as possíveis situações relacionadas às entradas e saídas. Contém as funções de pertinências das entradas e, para cada condição, as respectivas saídas. Essas regras ao serem aplicadas servem para aglutinar os resultados expressos em termos linguísticos. É nesse módulo onde se guardam as variáveis e suas classificações linguísticas. 
No processo de inferência (processamento) é usada a base de regras para a determinação dos termos linguísticos e um método de inferência, neste caso, o de Mamdani, de modo a inferir as ações de saída fuzzy, ou seja, determinar os respectivos graus de pertinência.

A implicação de Mamdani é definida por: $a \Rightarrow b \equiv a * \min b$, onde min é o "produto externo" correspondente à aplicação de $\min$ a cada elemento do produto cartesiano entre $a$ e $b$. Na prática, é equivalente à conjunção, ou seja, $a \min b$.

O processo de defuzzificação (saídas discretas) converte o conceito linguístico, resultado do processo de inferência, em um valor numérico utilizado como saída do controlador fuzzy. Para que esse processo ocorra, é preciso utilizar algum método de defuzzificação. Os métodos de defuzzificação mais utilizados são: método do centro da área, método centro dos máximos e método da média dos máximos. Neste estudo, o método utilizado foi o da média dos máximos, que produz um valor numérico que representa o valor médio de todos os cores ativados, conforme Equação 2.

$$
u_{m m}=\sum_{m=1}^{M} \frac{u_{m}}{M}
$$

onde , $u_{m m}=$ saída crisp $; u_{m}=$ core do termo linguístico $m ; M=$ quantidade de termos linguísticos ativados.

A utilização dos controladores fuzzy pretendeu fazer a calibragem dos resultados encontrados após as operações do Modelo COPPE-Cosenza, onde, através de uma base de regras, se consegue agrupar os vinte itens trabalhados em quatro fatores que definem as dimensões avaliadas no construto e contribuem de maneira mais coerente para se chegar a um resultado mais preciso na última etapa de mensuração da satisfação.

O programa utilizado para a determinação destes fatores por meio dos controladores fuzzy foi o Fuzzytech ${ }^{1}$, em sua versão livre, que processou as três etapas, ou seja, fuzzificação, inferência e defuzzificação.

O universo de discurso foi considerado como um intervalo de [0;1]. Os termos linguísticos determinados foram: muito insatisfeito, insatisfeito, indiferente, satisfeito e muito satisfeito.A função de pertinência utilizada no processo de fuzzificação foi a função triangular. O método de inferência selecionado foi o de Mamdani e o método de defuzzificação foi o método da média dos máximos, conforme mencionado anteriormente.

Depois de encontrados os valores atribuídos aos fatores, utilizou-se a Equação (3), definida na validação do instrumento desenvolvido por [5] para a determinação de um valor representativo da satisfação de cada indivíduo, sendo atribuído peso diferente a cada um dos fatores.

$$
\text { Nível de Satisfação }=0,71 f_{1}+0,12 f_{2}+0,10 f_{3}+0,07 f_{4} \text {, }
$$

onde $f_{1}, f_{2}, f_{3}$ e $f_{4}$ representam, respectivamente, atendimento, local de embarque/desembarque, veículo e segurança.

Os indivíduos foram denominados A, B e C. O cotejo para os 20 itens pertencentes ao instrumento foi realizado para os dois operados (matrizes de cotejo) apresentados nas Tabelas 8 e 9. Depois de encontrados os resultados, ditos índices fuzzy, foram utilizados os controladores fuzzy para atribuir um valor final aos quatro fatores, o que também foi executado por meio do Fuzzytech ${ }^{1}$. Ao analisar os resultados encontrados, percebeu-se que, em alguns casos, os valores encontrados para satisfação não estavam coerentes com as pontuações atribuídas aos itens (dados). A partir daí, foram analisadas algumas especificidades nos métodos das matrizes de cotejo, apresentadas nas Tabelas 8 e 9. Logo, percebeu-se a questão da avaliação dos indivíduos que consideram um determinado item desnecessário para sua satisfação, atribuindo zero para a demanda (ou avaliação da importância do item para a satisfação). Foram desconsiderados para este caso a regra II descrita acima, que diz que sempre que a oferta for maior que a demanda, o resultado considerado é o valor 1(um). Optou-se por desconsiderar o item sempre que sua demanda (importância) fosse considerada nula.

\footnotetext{
${ }^{1}$ Versão demo disponível em: http://www.fuzzytech.com/
} 
Com esta observação, refizeram-se os cálculos dos indivíduos A, B e C, obtendo-se uma maior coerência nos resultados encontrados. Com a conclusão do processo metodológico estabelecido, passou-se para a última fase que se forneceu a ordenação dos indivíduos em relação à satisfação.

\section{Resultados}

No primeiro processo de cotejo das matrizes "Diferença de Pertinências Adaptada" Tabela 8 e "Relacionamento de Pertinências Adaptada" Tabela 9 foram considerados os itens com valor de demanda nula. Os resultados encontrados para a satisfação e cada um dos quatro fatores que a mensuram são apresentados nas Tabelas 10, 11 e 12 .

Tabela 10: Resultados para fatores e satisfação por matriz de cotejo - Indivíduo A

\begin{tabular}{lccccc}
\hline Métodos adaptados & $f_{1}$ & $f_{2}$ & $f_{3}$ & $f_{4}$ & Satisfação \\
\hline Diferença & 0,70396 & 0,59998 & 0,82498 & 0,82498 & 0,7120 \\
Relacionamento & 0,52832 & 0,44116 & 0,76248 & 0,77352 & 0,5584 \\
\hline
\end{tabular}

Tabela 11:Resultados para fatores e satisfação por matriz de cotejo - indivíduo B

\begin{tabular}{lccccc}
\hline Métodos adaptados & $f_{1}$ & $f_{2}$ & $f_{3}$ & $f_{4}$ & Satisfação \\
\hline Diferença & 0,82500 & 0,95000 & 0,17498 & 0,59998 & 0,75925 \\
Relacionamento & 0,82500 & 0,95000 & 0,17489 & 0,59998 & 0,75925 \\
\hline
\end{tabular}

Tabela 12:Resultados para fatores e satisfação por matriz de cotejo - indivíduo C

\begin{tabular}{lccccc}
\hline Métodos adaptados & $f_{1}$ & $f_{2}$ & $f_{3}$ & $f_{4}$ & Satisfação \\
\hline Diferença & 0,95000 & 0,95000 & 0,95000 & 0,95000 & 0,95000 \\
Relacionamento & 0,95000 & 0,95000 & 0,95000 & 0,95000 & 0,95000 \\
\hline
\end{tabular}

Fazendo uma análise geral dos valores respondidos pelos indivíduos $\mathrm{A}, \mathrm{B}$ e $\mathrm{C}$ percebeu-se que os valores encontrados nos indivíduos $\mathrm{A}, \mathrm{B}$ e $\mathrm{C}$, que continham valores de demanda igual à zero não eram muito coerentes com os dados apresentados. Logo, com o procedimento mencionado anteriormente, que desconsidera estes itens, os valores encontrados estão apresentados nas Tabelas 13, 14 e 15.

Tabela 13: Resultados para fatores e satisfação por operador, desconsiderando demanda nula - indivíduo A

\begin{tabular}{lccccc}
\hline Métodos adaptados & $f_{1}$ & $f_{2}$ & $f_{3}$ & $f_{4}$ & Satisfação \\
\hline Diferença & 0,60280 & 0,59998 & 0,82498 & 0,82498 & 0,64023 \\
Relacionamento & 0,34372 & 0,44116 & 0,76248 & 0,77352 & 0,42737 \\
\hline
\end{tabular}

Tabela 14:Resultados para fatores e satisfação por operador, desconsiderando demanda nula - indivíduo B

\begin{tabular}{lccccc}
\hline Métodos adaptados & $f_{1}$ & $f_{2}$ & $f_{3}$ & $f_{4}$ & Satisfação \\
\hline Diferença & 0,71668 & 0,95000 & 0,17498 & 0,59998 & 0,68233 \\
Relacionamento & 0,71668 & 0,95000 & 0,17489 & 0,59998 & 0,68233 \\
\hline
\end{tabular}

Tabela 15:Resultados para fatores e satisfação por operador, desconsiderando demanda nula -indivíduo C

\begin{tabular}{lccccc}
\hline Métodos adaptados & $f_{1}$ & $f_{2}$ & $f_{3}$ & $f_{4}$ & Satisfação \\
\hline Diferença & 0,95000 & 0,95000 & 0,95000 & - & 0,88350 \\
Relacionamento & 0,95000 & 0,95000 & 0,95000 & - & 0,88350 \\
\hline
\end{tabular}

Organizando os dados e estabelecendo uma hierarquia entre os escores finais, obtém-se as informações apresentadas na Tabela 16 . 
Tabela 16: Resultado final dos indivíduos A, B e C para satisfação

\begin{tabular}{ccc}
\hline \multirow{2}{*}{ Indivíduo } & \multicolumn{2}{c}{ Métodos adaptados } \\
& Diferença & Relacionamento \\
\hline A & 0,64023 & 0,42737 \\
B & 0,68233 & 0,68233 \\
C & 0,88350 & 0,88350 \\
\hline
\end{tabular}

Com base nos resultados da Tabela 16, percebe-se que ambas as matrizes de cotejo proporcionaram a mesma ordem para o nível de satisfação dos indivíduos. O indivíduo $\mathrm{C}$ foi o mais satisfeito, o indivíduo $\mathrm{B}$ foi o intermediário e o indivíduo A foi o menos satisfeito. Estes resultados, de maneira geral, são aceitáveis quando se observa o banco de dados utilizado. Ainda, comparando os dois resultados, verifica-se que entre as duas matrizes de cotejo, os valores para os indivíduos $\mathrm{B}$ e $\mathrm{C}$ foram idênticos e para o indivíduo $\mathrm{A}$, os valores diferiram entre os dois procedimentos operacionais. Porém, esta diferença não influencia na hierarquia entre os indivíduos quanto ao nível de satisfação. No entanto, compreende-se que estas diferenças podem ser encontradas com mais frequência em uma maior amostra do banco de dados, o que ainda deve ser estudado para ser possível afirmar que a utilização de diferentes matrizes de cotejo não influenciará o ranking de pessoas mais ou menos satisfeitas.

\section{Resultados}

O presente trabalho mostra a adaptação de um modelo de análise hierárquica para a mensurar a satisfação utilizando a lógica fuzzy. Acredita-se que a adaptação do modelo proposto pode ser um instrumento de fácil compreensão e manuseio, além de possibilitar resultados bastante eficientes na avaliação de variáveis subjetivas como a satisfação. Considera-se ainda que poderá ser útil na elaboração de estratégias e avaliações empresariais, dentre outras possibilidades que envolvam variáveis desta natureza.

Este trabalho apresenta os resultados de um estudo piloto sobre o funcionamento de uma adaptação do modelo COPPE-Cosenza Fuzzy, etapa inicial de um estudo mais amplo. Outras análises estão sendo realizadas para comprovar a sua provável real eficácia.

\section{Agradecimentos}

Agradecemos à CAPES pelo auxílio financeiro em forma de Bolsa de Mestrado - Demanda Social, à Universidade Federal do Rio Grande e ao Programa de Pós-graduação em Modelagem Computacional.

\section{Referências}

[1] ZADEH, L. A., Fuzzy Sets. Information And Control, v. 8, Iss. 3, pgs 338-353, 1965. Disponível em: $<$ http://www.sciencedirect.com/science/article/pii/S001999586590241X> Acesso em: 10 de fev. de 2016.

[2] Clemente A. Projetos Empresariais e Públicos. 2 Ed. São Paulo: Atlas, 2002.

[3] FIUZA, S. N. et al. Localização industrial na Região Norte Fluminense: uma análise em termos de oferta e demanda por fatores locacionais. Revista Brasileira de Gestão e Desenvolvimento Regional, v. 8, n. 3, pp. 198-238, 2012. Disponível em: $<$ https://www.researchgate.net/profile/Henrique_Da_Hora/publication/233819409_Localizacao_industrial_ na_Regiao_Norte_Fluminense_uma_analise_em_termos_de_oferta_e_demanda_por_fatores_locacionais/li $\mathrm{nks} / 0912 \mathrm{f} 5 \overline{1} 10069 \overline{5} \mathrm{e} 8 \mathrm{da} 4 \mathrm{e} 000000 . \mathrm{pdf}>$ Acesso em: $10 \mathrm{de}$ fev. de $20 \overline{1} \overline{6}$.

[4] MARTINS, G. W., Uma Contribuição aos estudos de localização industrial: Determinando o potencial de transporte aéreo de uma região com base no modelo de análise hierárquica COPPE-Cosenza. Tese de Doutorado. Universidade Federal do Rio de Janeiro, RJ, 2010. Disponível em: $<$ http://objdig.ufrj.br/60/teses/coppe_m/GuilhermeWeberMartins.pdf> Acesso em: 10 de fev. de 2016. 
[5] TOLEDO, O. M., Um caso de aplicação da Lógica Fuzzy - O Modelo Coppe-Cosenza de Hierarquia Fuzzy. XXIII Encontro Nacional de Engenharia de Produção-Anais, v. 1, 2003. Disponível em: $<$ http://www.boente.eti.br/fuzzy/paper-fuzzy-cosenza.pdf $>$. Acesso em: 10 de fev. de 2016.

[6] CHAMOVITZ I.; COSENZA C. A. N., Aplicação do Modelo de Hierarquia Fuzzy COPPE-Cosenza para a Avaliação de Grupos Operativos em Fóruns Educacionais na Internet. Tese de Doutorado, Programa de Pós-Graduação em Engenharia de Produção, COPPE Universidade Federal do Rio de Janeiro, RJ, 2010. Disponível

em: <http://www.academia.edu/2242335/Aplica\%C3\%A7\%C3\%A3o_do_modelo_de_hierarquia_fuzzy_COPP E-

COSENZA_para_a_avalia\%C3\%A7\%C3\%A3o_de_Grupos_Operativos_em_f $\% \mathrm{C} 3 \% \mathrm{~B} 3$ runs_educacionais _na_Internet>. Acesso em: $10 \mathrm{de}$ fev. de 2016.

[7] MATTOS, V. L. D.; AVILA, S. H. ; FIGUEIREDO, V. L. M. Um instrumento para medir a satisfação dos passageiros de um sistema de transporte público. GEPROS. Gestão da Produção, Operações e Sistemas (Online), 2016.

[8] AFFONSO, C. R. Aplicação de Lógica Fuzzy à Localização de Instalações.Tese de Doutorado. Universidade Federal do Rio de Janeiro, 2012. Disponível em: $<$ http://monografias.poli.ufrj.br/monografias/monopoli10004897.pdf $>$. Acesso em: 10 de fev. de 2016. 